\title{
Determination of Electroweak Parameters using H1 Inclusive Deep Inelastic Scattering Data
}

\section{Zhiqing Zhang ${ }^{* i}$}

LAL, Univ. Paris-Sud, CNRS/IN2P3, Université Paris-Saclay, Orsay, France

E-mail: zhangzq@lal.in2p3.fr

\begin{abstract}
An improved determination of electroweak parameters using $\mathrm{H} 1$ inclusive neutral and charged current deep inelastic scattering cross section data is presented. The analysis benefits from the use of the previously published data with longitudinally polarised lepton beams. The parameters are determined in a combined fit of the electroweak parameters together with PDFs. The predictions include NNLO QCD corrections for the PDF and structure function calculations, and the corrections at the leptonic vertex are obtained in the on-shell scheme including the full set of oneloop corrections. The analysis determines the weak neutral-current couplings of the light quarks as well as potential contributions beyond the SM. The mass of the W-boson is determined with a precision of $115 \mathrm{MeV}$ in the space-like regime.
\end{abstract}

The 39th International Conference on High Energy Physics (ICHEP2018)

4-11 July, 2018

Seoul, Korea

${ }^{*}$ Speaker.

$\dagger$ on behalf of the H1 Collaboration. This proceedings is a shortened version of that of DIS2018. 
The deep inelastic scattering (DIS) of leptons off nucleons has played an important role in revealing the structure of matter, in the discovery of weak neutral current interactions and in the foundation of the Standard Model (SM) as the theory of strong and electroweak (EW) interactions. At HERA, electrons (or positrons) of $27.6 \mathrm{GeV}$ were colliding with protons of up to $920 \mathrm{GeV}$ in the first data taking period, HERA-I, from 1993 to 2000 with unpolarised lepton beams, and in the second period, HERA-II, from 2003 to 2007 with the longitudinally polarised lepton beams, $P_{e}$, to a level between $-37 \%$ and $36 \%$. In addition, the integrated luminosity value of the $e^{+} p$ and $e^{-} p$ collisions at HERA-II has increased by a factor of 1.5 and 15 over that at HERA-I, respectively. Both neutral current (NC) and charged current (CC) interactions occur in ep collisions and a large set of precise NC and CC cross sections was measured by the $\mathrm{H} 1$ experiment. In terms of $Q^{2}$, the negative four-momentum transfer squared, the kinematic coverage includes the region where the weak and electromagnetic interactions become of comparable strength.

A first determination of EW parameters including the vector and axial-vector weak neutral couplings of the light quarks, $q=u, d$, to the $Z$-boson was performed using HERA-I H1 data [1]. In this proceedings, we present the final results from $\mathrm{H} 1$ by including $\mathrm{NC}$ and $\mathrm{CC}$ cross section data from HERA-II [2].

NC interactions in the process $e^{ \pm} p \rightarrow e^{ \pm} X$, with $X$ standing for any hadronic final state, are mediated by a virtual photon $(\gamma)$ or $Z$ boson in the $t$-channel, and the cross section is expressed in terms of generalised structure functions $\tilde{F}_{2}^{ \pm}, x \tilde{F}_{3}^{ \pm}$and $\tilde{F}_{\mathrm{L}}^{ \pm}$at EW leading order (LO) as $\frac{d^{2} \sigma^{\mathrm{NC}}\left(e^{ \pm} p\right)}{d x d Q^{2}}=\frac{2 \pi \alpha^{2}}{x Q^{4}}\left[Y_{+} \tilde{F}_{2}^{ \pm}\left(x, Q^{2}\right) \mp Y_{-} x \tilde{F}_{3}^{ \pm}\left(x, Q^{2}\right)-y^{2} \tilde{F}_{\mathrm{L}}^{ \pm}\left(x, Q^{2}\right)\right]$, where $\alpha$ is the fine structure constant and $x$ denotes the Bjorken scaling variable. The helicity dependence of the interaction is contained in the terms $Y_{ \pm}=1 \pm(1-y)^{2}$ with $y$ being the inelasticity of the scattering process. The generalised structure functions can be separated into contributions from pure $\gamma$ - and $Z$ exchange and their interference, $\tilde{F}_{2}^{ \pm}=F_{2}-\left(g_{V}^{e} \pm P_{e} g_{A}^{e}\right) \varkappa_{Z} F_{2}^{\gamma Z}+\left[\left(g_{V}^{e} g_{V}^{e}+g_{A}^{e} g_{A}^{2}\right) \pm 2 P_{e} g_{V}^{e} g_{A}^{e}\right] \varkappa_{Z}^{2} F_{2}^{Z}$, $\tilde{F}_{3}^{ \pm}=-\left(g_{A}^{e} \pm P_{e} g_{V}^{e}\right) \varkappa_{Z} F_{3}^{\gamma Z}+\left[2 g_{V}^{e} g_{A}^{e} \pm P_{e}\left(g_{V}^{e} g_{V}^{e}+g_{A}^{e} g_{A}^{e}\right)\right] \varkappa_{Z}^{2} F_{3}^{Z}$, and similarly for $\tilde{F}_{L}$. The variables $g_{A}^{e}$ and $g_{V}^{e}$ stand for the axial-vector and vector couplings of the lepton $e^{ \pm}$to the $Z$ boson, respectively. The $Q^{2}$-dependent coefficient $\varkappa_{Z}$ accounts for the $Z$-boson propagator, $\varkappa_{Z}\left(Q^{2}\right)=\frac{Q^{2}}{Q^{2}+m_{Z}^{2}}$ $\frac{1}{4 \sin ^{2} \theta_{W} \cos ^{2} \theta_{W}}=\frac{Q^{2}}{Q^{2}+m_{Z}^{2}} \frac{G_{\mathrm{F}} m_{Z}^{2}}{2 \sqrt{2} \pi \alpha}$. It can be normalised using the weak mixing angle, $\sin ^{2} \theta_{W}=1-$ $m_{W}^{2} / m_{Z}^{2}$, i.e. using the $W$ and $Z$ boson masses, $m_{W}$ and $m_{Z}$, or the Fermi coupling constant $G_{\mathrm{F}}$. The structure functions are related to linear combinations of the quark and anti-quark momentum distributions, $x q$ and $x \bar{q}$. For instance, the $F_{2}$ and $x F_{3}$ structure functions in the naive quark-parton model, i.e. at LO in QCD, are: $\left[F_{2}, F_{2}^{\gamma Z}, F_{2}^{Z}\right]=x \sum_{q}\left[Q_{q}^{2}, 2 Q_{q} g_{V}^{q}, g_{V}^{q} g_{V}^{q}+g_{A}^{q} g_{A}^{q}\right]\{q+\bar{q}\}, x\left[F_{3}^{\gamma Z}, F_{3}^{Z}\right]=$ $x \sum_{q}\left[2 Q_{q} g_{A}^{q}, 2 g_{A}^{q} g_{A}^{q}\right]\{q-\bar{q}\}$. The axial-vector and vector couplings of the quarks $q$ to the $Z$ boson, $g_{A}^{q}$ and $g_{V}^{q}$, depend on the electric charge, $Q_{q}$, in units of the positron charge, and on the third component of the weak-isospin of the quarks, $I_{\mathrm{L}, q}^{(3)}$. In terms of $\sin ^{2} \theta_{W}$, they are given by the standard EW theory:

$$
g_{A}^{q}=\sqrt{\rho_{\mathrm{NC}, q}} I_{\mathrm{L}, q}^{(3)}, \quad g_{V}^{q}=\sqrt{\rho_{\mathrm{NC}, q}}\left(I_{\mathrm{L}, q}^{(3)}-2 Q_{q} \kappa_{\mathrm{NC}, q} \sin ^{2} \theta_{W}\right),
$$

where the $Q^{2}$-dependent form factors $\rho_{\mathrm{NC}, q}$ and $\kappa_{\mathrm{NC}, q}$ account for universal higher-order corrections [3]. The same formulae also apply to the lepton couplings $g_{A / V}^{e}$.

The $\mathrm{CC}$ cross section at $\mathrm{LO}$ is written as $\frac{d^{2} \sigma^{\mathrm{CC}}\left(e^{ \pm} p\right)}{d x d Q^{2}}=\left(1 \pm P_{e}\right) \frac{G_{\mathrm{F}}^{2}}{4 \pi x}\left[\frac{m_{W}^{2}}{m_{W}^{2}+Q^{2}}\right]^{2}$ 
$\left(Y_{+} W_{2}^{ \pm}\left(x, Q^{2}\right) \mp Y_{-} x W_{3}^{ \pm}\left(x, Q^{2}\right)-y^{2} W_{\mathrm{L}}^{ \pm}\left(x, Q^{2}\right)\right) . \quad$ In the quark-parton model, $W_{\mathrm{L}}^{ \pm}=0$, and the structure functions $W_{2}^{ \pm}$and $x W_{3}^{ \pm}$are obtained from the parton distribution functions. For electron scattering, only positively charged quarks contribute:

$$
W_{2}^{-}=x\left(\rho_{\mathrm{CC}, e q}^{2} U+\rho_{\mathrm{CC}, e \bar{q}}^{2} \bar{D}\right), \quad x W_{3}^{-}=x\left(\rho_{\mathrm{CC}, e q}^{2} U-\rho_{\mathrm{CC}, e \bar{q}}^{2} \bar{D}\right),
$$

while negatively charged quarks contribute to positron scattering:

$$
W_{2}^{+}=x\left(\rho_{\mathrm{CC}, e q}^{2} \bar{U}+\rho_{\mathrm{CC}, e \bar{q}}^{2} D\right), \quad x W_{3}^{+}=x\left(\rho_{\mathrm{CC}, e \bar{q}}^{2} D-\rho_{\mathrm{CC}, e q}^{2} \bar{U}\right),
$$

where higher-order EW corrections are collected in form factors $\rho_{\mathrm{CC}, e q / e \bar{q}}$. Below the top-quark threshold, one has $U=u+c, \bar{U}=\bar{u}+\bar{c}, D=d+s, \bar{D}=\bar{d}+\bar{s}$.

In the on-shell (OS) scheme, the independent parameters of the SM EW theory are determined by the fine structure constant $\alpha$ and the masses of the gauge bosons, the Higgs boson $m_{H}$, and the fermions $m_{f}$. The weak mixing angle is then fixed, and $G_{\mathrm{F}}$ is a prediction, given by $G_{\mathrm{F}}=\frac{\pi \alpha}{\sqrt{2} m_{W}^{2}} \frac{1}{\sin ^{2} \theta_{W}} \frac{1}{(1-\Delta r)}$, where higher-order corrections enter through the quantity $\Delta r=\Delta r\left(\alpha, m_{W}, m_{Z}, m_{H}, m_{t}, \ldots\right)[4]$.

The analysis is performed at next-to-next-to-leading order (NNLO) in QCD including oneloop EW correction [2]. The DGLAP formalism [5] is used to describe the evolution of the PDFs with $Q^{2}$. The PDFs are parameterised with 13 parameters at a starting scale of $1.9 \mathrm{GeV}^{2}$ following Ref. [6]. The EW parameters are determined together with PDF parameters in combined EW and QCD fits to all NC and CC cross section data from HERA-I and HERA-II measured by H1. The $\mathrm{PDF}$ fit alone, i.e. all EW parameters set to their SM values [3], yields a fit quality of $\chi^{2}$ per degree of freedom of 1.03 .

In one fit, $m_{W}$ is treated as a free parameter, it is determined together with the PDF parameters with the result $m_{W}=80.502 \pm 0.070_{\text {stat }} \pm 0.055_{\text {syst }} \pm 0.074_{\mathrm{PDF}}=80.520 \pm 0.115_{\text {total }} \mathrm{GeV}$. The total uncertainty is improved by a factor of two in comparison to earlier result based on HERA-I data only. This $W$-mass determination in the space-like regime is found to be consistent with other single experiments [7] as well as with the world average value of $80.385 \pm 0.015 \mathrm{GeV}$ [3].

For the determination of the weak NC couplings of the quarks to the $Z$ boson, the couplings are treated as free parameters together with the PDF parameters. The precision of the vector couplings is significantly improved as compared to that of HERA-I [1], mainly due to the polarised lepton beams at HERA-II. The H1 results are compared in figure 1 (left) to those from LEP+SLC [8] and D0 [9] at Tevatron. The different contour shapes, particularly for the $u$-type quarks, show the potential to achieve an ultimate precision if they would be combined, in particular since the HERA result does not exhibit sign ambiguities or ambiguities between axial-vector and vector couplings, which are for example present in determinations from $Z$-decays at the pole.

Additional fits are performed allowing deviations from the SM predictions by replacing form factors in equations (1), (2) and (3) with $\rho_{\mathrm{NC}, q} \rightarrow \rho_{\mathrm{NC}, q}^{\prime} \rho_{\mathrm{NC}, q}, \kappa_{\mathrm{NC}, q} \rightarrow \kappa_{\mathrm{NC}, q}^{\prime} \kappa_{\mathrm{NC}, q}, \rho_{\mathrm{CC}, e q / e \bar{q}} \rightarrow$ $\rho_{\mathrm{CC}, e q / e \bar{q}}^{\prime} \rho_{\mathrm{CC}, e q / e \bar{q}}$. The results for the $\mathrm{NC}$ channel are shown in figure 1 (middle). The results are consistent with the SM predictions in $1-2$ standard deviations.

Potential scale dependence of these BSM form factors are further checked by performing alternative fits in four $Q^{2}$ bins with a common set of PDF parameters. The CC study (figure 1 (right)) represents the first determination of the $\rho_{\mathrm{CC}, e q / e \bar{q}}^{\prime}$ parameters for separate quark flavours and also its first scale dependence test. 

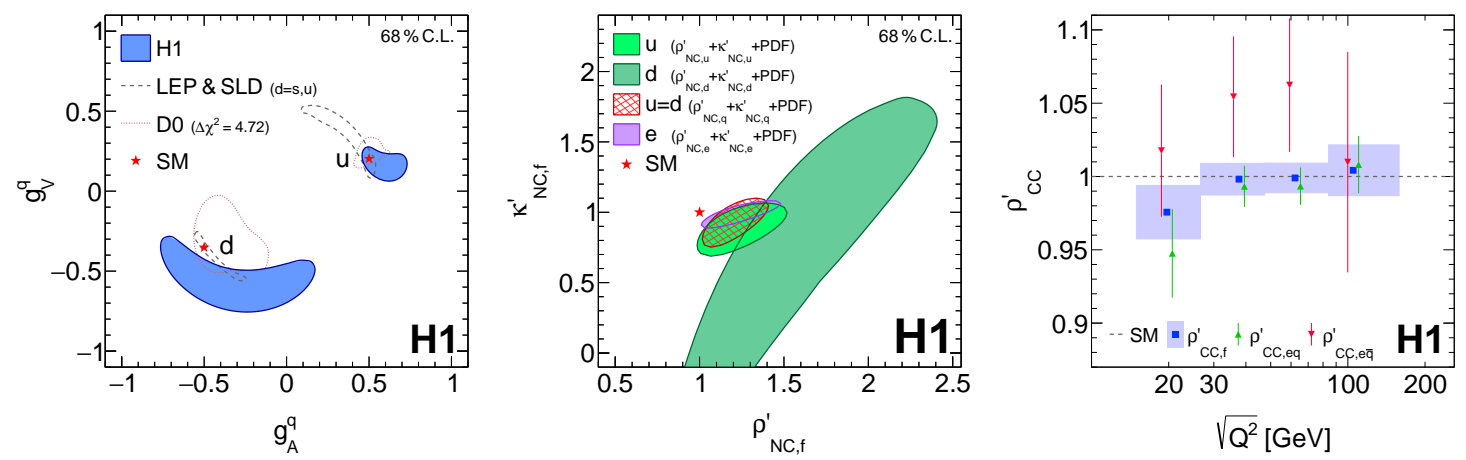

Figure 1: Left: Weak NC couplings of the $u$ - and $d$-type quarks at the $68 \%$ confidence level (C.L.). Middle: Parameters $\rho_{\mathrm{NC}, f}^{\prime}$ and $\kappa_{\mathrm{NC}, f}^{\prime}$ for $u$ - and $d$-type quarks and electrons at $68 \%$ C.L.. Right: Parameters $\rho_{\mathrm{CC}}^{\prime}$ determined for four different values of $Q^{2}$. The error bars, as well as the height of the shaded areas, indicate the total uncertainties of the measurement. The width of the shaded areas indicates the $Q^{2}$ range probed by the selected data. The values for the $\rho_{\mathrm{CC}, e q}^{\prime}$ and $\rho_{\mathrm{CC}, e \bar{q}}^{\prime}$ parameters are horizontally displaced for better visibility. The plots are taken from [2].

To conclude, the inclusive deep-inelastic scattering cross section data measured by $\mathrm{H} 1$ at HERA-II with the longitudinally polarised lepton beams and higher integrated luminosity have provided additional sensitivity for improving the precision of the electroweak parameters. The $Q^{2}$ dependence of the $\mathrm{H} 1$ data allows for a study of the scale dependence of the $\rho_{\mathrm{NC}}^{\prime}, \kappa_{\mathrm{NC}}^{\prime}$ and $\rho_{\mathrm{CC}}^{\prime}$ parameters in the range $12<\sqrt{Q^{2}}<100 \mathrm{GeV}$.

\section{References}

[1] H1 Collaboration (A. Aktas et al.), Phys. Lett. B 632 (2006) 35 [hep-ex/0507080].

[2] H1 Collaboration (V. Andreev et al.) and H. Spiesberger, Eur. Phys. J. C 78 (2018) 777 [arXiv:1806.01176].

[3] Particle Data Group (C. Patrignani et al.), Chin. Phys. C 40 (2016) 10.

[4] A. Sirlin, Phys. Rev. D 22 (1980) 971.

[5] Y.L. Dokshitzer, Sov. Phys. JETP 46, 641 (1977); V.N. Gribov and L.N. Lipatov, Sov. J. Nucl. Phys. 15, 675 (1972); V.N. Gribov and L.N. Lipatov, Sov. J. Nucl. Phys. 15, 438 (1972); G. Altarelli and G. Parisi, Nucl. Phys. B 126, 298 (1977).

[6] H1 Collaboration (F.D. Aaron et al.), JHEP 09, 061 (2012) [arXiv:1206.7007].

[7] ALEPH Collaboration (R. Barate et al.), Phys. Lett. B 415 (1997) 435; L3 Collaboration (M. Acciarri et al.), Phys. Lett. B 407 (1997) 419; OPAL Collaboration (K. Ackerstaff et al.), Phys. Lett. B 389 (1996) 416; DELPHI Collaboration (J. Abdallah et al.), Eur. Phys. J. C 55 (2008) 1 [arXiv:0803.2534]; D0 Collaboration (V.M. Abazov et al.), Phys. Rev. Lett. 108 (2012) 151804 [arXiv:1203.0293]; CDF Collaboration (T. Aaltonen et al.), Phys. Rev. Lett. 108 (2012) 151803 [arXiv:1203.0275]; CDF Collaboration (T.A. Aaltonen et al.), Phys. Rev. D 89 (2014) 072003 [arXiv:1311.0894]; ATLAS Collaboration, Eur. Phys. J. C 78 (2018) 110 [arXiv:1701.07240].

[8] LEP and SLD Collaborations (S. Schael et al.), Phys. Rept. 427 (2006) 257 [hep-ex/0509008].

[9] D0 Collaboration (V.M. Abazov et al.) Phys. Rev. D 84 (2011) 012007 [arXiv:1104.4590]. 